\section{Liver impaction technique to prevent shearing of the guidewire during endoscopic ultrasound- guided hepaticogastrostomy}

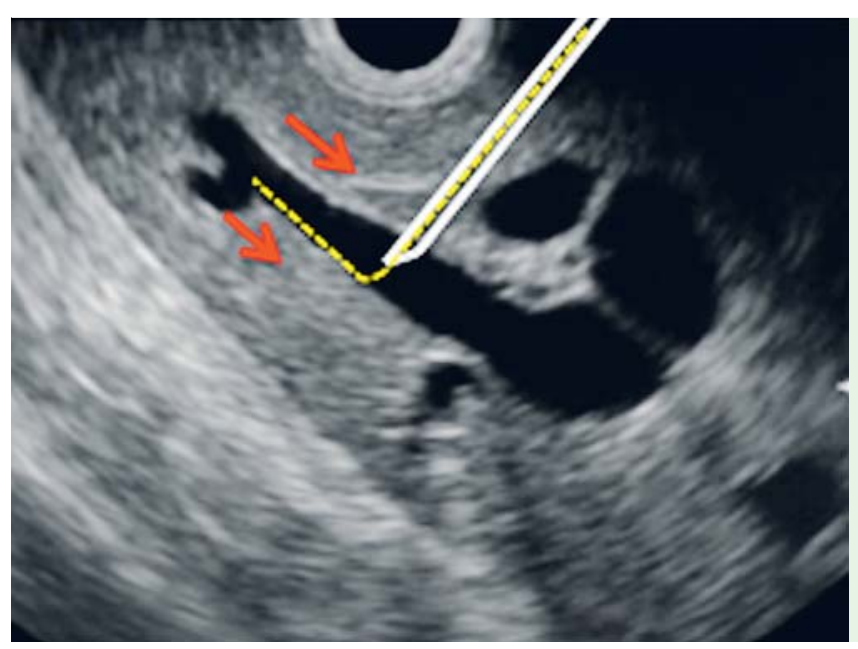

Fig. 1 A 79-year-old man was undergoing endoscopic ultrasoundguided hepaticogastrostomy (EUS-HG). The guidewire (yellow dashed line) was accidentally advanced into a peripheral bile duct and therefore needed to be retracted (red arrows) into the fineneedle aspiration needle, before re-advancement into the hepatic hilar duct.

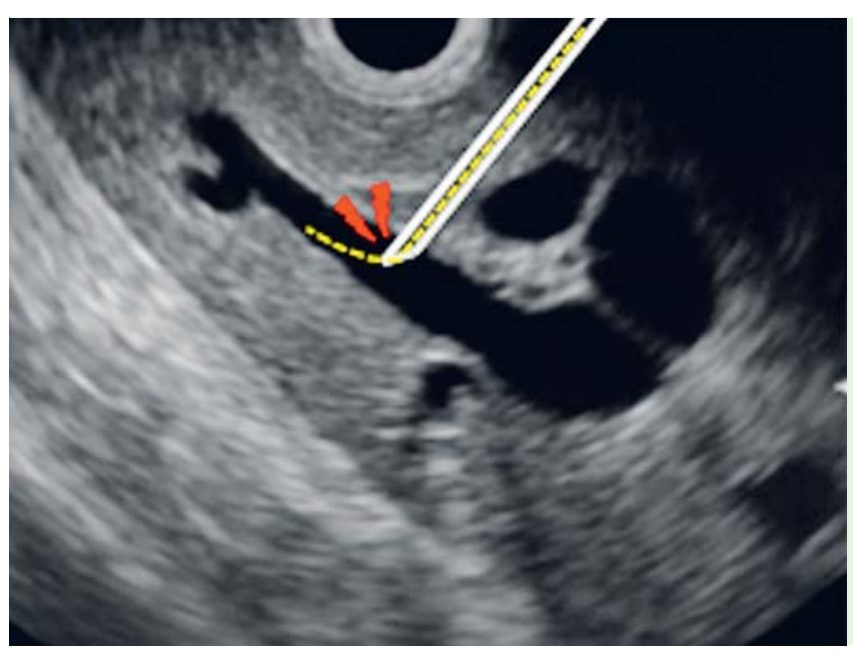

Fig. 2 When the guidewire (yellow dashed line) is pulled back, it may be sheared (red arrowheads) by the tip of the needle.

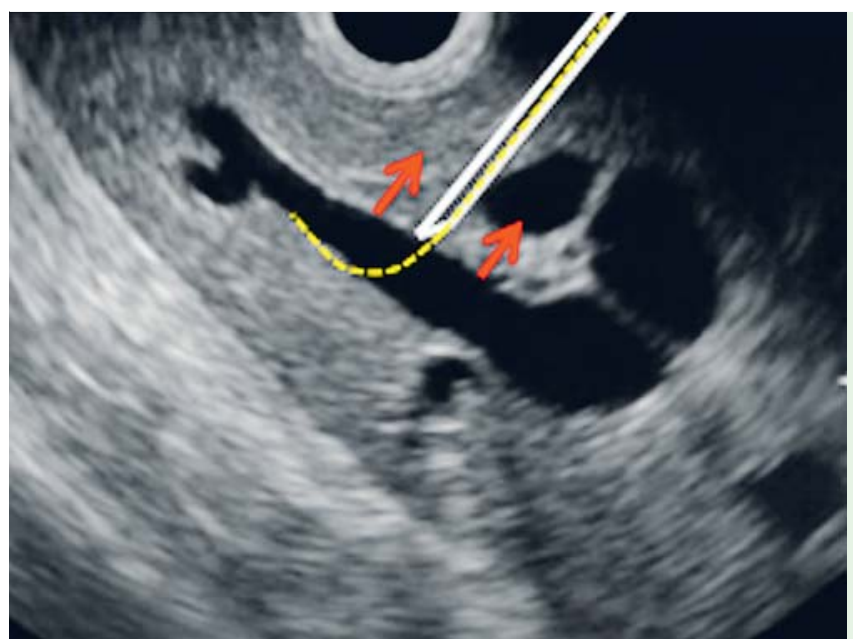

During endoscopic ultrasound-guided hepaticogastrostomy (EUS-HG) [1,2], the intrahepatic bile duct is small in diameter (usually 2-5 mm), with various angles or curves between it and the common bile duct. If the guidewire is advanced into a peripheral bile duct ( $\bullet$ Fig. 1 ), the guidewire must be retracted and advanced again. However, shearing of the guidewire may occur when it is pulled into the fineneedle aspiration (FNA) needle ( $\bullet$ Fig. 2 ). If this adverse event arises, we use the "liver impaction technique." First, the guidewire is pushed adequately into the peripheral bile duct, and the FNA needle is pulled back into the hepatic parenchyma ( Fig.3). Because the tip of the FNA needle is then within the hepatic parenchyma, shearing becomes less likely (๑ Fig.4).

A 79-year-old man was admitted to our hospital with obstructive jaundice due to advanced pancreatic cancer with duodenal obstruction. First, the left intrahepatic bile duct was punctured with a 19-gauge FNA needle. Contrast medium was injected. Although a 0.025-inch guidewire was inserted, it was accidentally introduced into a peripheral bile duct. We retracted the guidewire to advance it into the common bile duct or hepatic hilar duct. However, shearing of the guidewire occurred. After the guidewire had been pushed into the peripheral bile duct, we carefully pulled the FNA needle into the hepatic parenchyma with EUS imaging guidance. We were then easily able to pull the guidewire back into the FNA needle. We then successfully re-advanced the guidewire, into the bile duct in the hepatic hilum. After the FNA needle had been exchanged for an endoscopic retrograde cholangiopancreatography (ERCP) catheter, contrast medium was injected, and obstruction was seen in the lower bile duct. Finally, we performed EUS-HG to completion ( Video 1 ). No adverse events were associated with this procedure.

Our technique may be useful to prevent guidewire shearing.

Endoscopy_UCTN_Code_TTT_1AS_2AD 


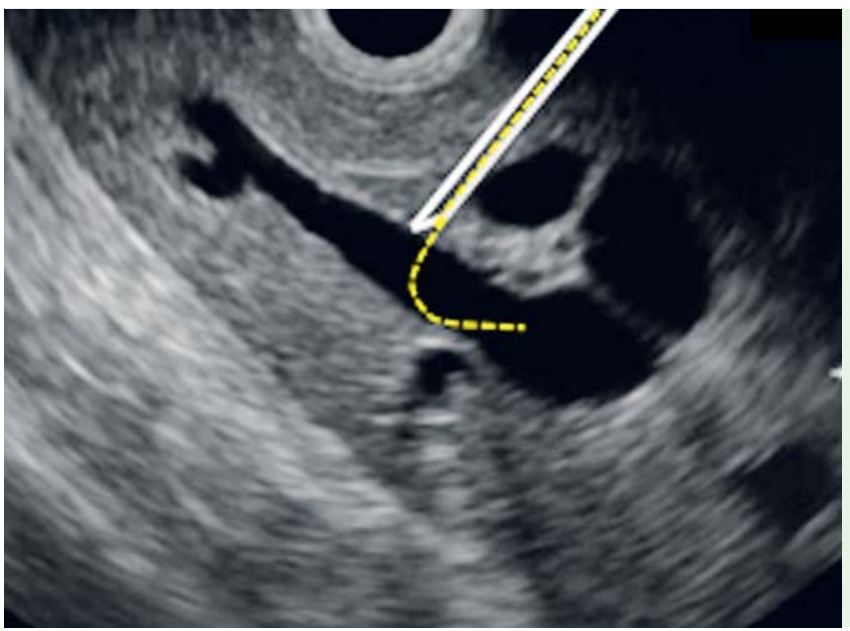

Fig. 4 Because the tip of the fine-needle aspiration (FNA) needle is within the hepatic parenchyma, shearing between the guidewire and the FNA needle is now unlikely to occur.

\section{Competing interests: None}

Takeshi Ogura, Daisuke Masuda, Toshihisa Takeuchi, Shinya Fukunishi, Kazuhide Higuchi

2nd Department of Internal Medicine, Osaka Medical College, Osaka, Japan

\section{References}

1 Ogura T, Kurisu Y, Masuda D et al. Novel method of endoscopic ultrasound-guided hepaticogastrostomy to prevent stent dysfunction. J Gastroenterol Hepatol 2014; 29: $1615-1621$

2 Itoi T, Isayama $H$, Sofuni A et al. Stent selection and tips on placement technique of EUS-guided biliary drainage: transduodenal and transgastric stenting. J Hepatobiliary Pancreat Sci 2011; 18: 664-672
Bibliography Dol http://dx.doi.org/ 10.1055/s-0034-1393381 Endoscopy 2015; 47: E583-E584

(c) Georg Thieme Verlag KG

Stuttgart · New York ISSN 0013-726X

Corresponding author Takeshi Ogura, MD

2nd Department of Internal Medicine Osaka Medical College

2-7 Daigakuchou

Takatsukishi

Osaka 569-8686

Japan

Fax: $+81-726846532$

oguratakeshi0411@yahoo.co.jp

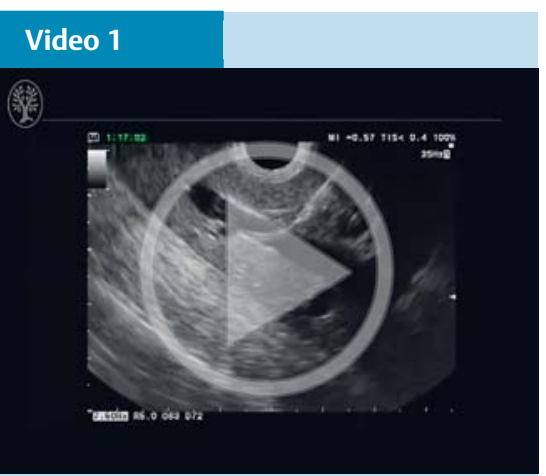

During endoscopic ultrasound-guided hepaticogastrostomy (EUS-HG), the guidewire is inadvertently introduced into a peripheral intrahepatic bile duct and then, upon retraction, shears against the fine-needle aspiration (FNA) needle. Therefore, after pushing the guidewire into the peripheral bile duct, we carefully pull the FNA needle back into the hepatic parenchyma under EUS guidance. The guidewire is then easily retracted into the FNA needle without shearing, and then successfully re-advanced into the bile duct in the hepatic hilum. Finally, EUS-guided hepaticogastrostomy is completed. 